Check for updates

Cite this: RSC Adv., 2017, 7, 41763

Received 20th May 2017

Accepted 29th July 2017

DOI: $10.1039 / c 7 r a 05697 d$

rsc.li/rsc-advances

\title{
Larvicidal, super hydrophobic and antibacterial properties of herbal nanoparticles from Acalypha indica for biomedical applications $\dagger$
}

\author{
Karthik S., (D) ${ }^{a}$ Suriyaprabha R., (D) ${ }^{a}$ Vinoth M., ${ }^{a}$ Srither S. R., ${ }^{a}$ Manivasakan P., \\ Rajendran V. (DD *a and Suresh Valiyaveettil (D) ${ }^{\text {b }}$
}

\begin{abstract}
The present study is aimed at developing a biocompatible nanomaterial with excellent medicinal properties using herbs. The herbal nanoparticles were prepared from shade dried leaves of Acalypha indica using the ball-milling technique. The prepared nanoparticles were characterized using X-ray diffraction, Fourier transform infrared spectroscopy, ultraviolet-visible spectroscopy, particle size analysis, scanning electron microscopy, X-ray fluorescence spectroscopy and transmission electron microscopy. The amorphous herbal AINPs posses an average particle size distribution of $54 \pm 3 \mathrm{~nm}$ and a UV-absorption maximum at $434 \mathrm{~nm}$, and are superhydrophobic $\left(151^{\circ}\right)$ in nature. The prepared herbal AINPs were tested for their antimicrobial properties against Staphylococcus aureus and Escherichia coli. Mosquito repellent properties were investigated against three disease vectors, namely, Aedes aegypti, Anopheles stephensi and Culex quinquefasciatus, and showed significant larvicidal activity due to the existence of phytochemical compounds in the herbal nanoparticles. The acute toxicity of the herbal nanoparticles was tested with an in vivo animal model, zebrafish (Danio rerio), to ensure biocompatibility. The observed results confirmed that herbal AINPs play a dominant role in enhancing the medicinal properties for different biomedical applications.
\end{abstract}

\section{Introduction}

Everyday human life is affected by skin infections and mosquito related diseases such as boils, impetigo, cellulitis, malaria, dengue, lymphatic filariasis and yellow fever. ${ }^{1}$ Recent developments in nanotechnology demonstrate the practical applications of nanoparticles from plant extracts, due to their enhanced antibacterial, antifungal, hydrophobic, mosquito repellent and UV protection properties. ${ }^{2}$ Many natural products are widely used to develop newer medicines with potent biological and pharmacological activities. Medicinal plant based drugs have been extensively used in skin allograft, cornea allograft, intestinal allograft, cardiac allograft, and in the treatment of various diseases such as malaria, symptomatic endometriosis, uterine adenomyosis, leiomyoma, etc. ${ }^{3-5}$ The herbal nanoparticle treated materials are known in the field of biomedical applications for their exotic properties, e.g., non-toxic, mosquito repellent, hydrophobic, ultraviolet protection (UV) and antimicrobial action. ${ }^{6-9}$

${ }^{a}$ Centre for Nano Science and Technology, K. S. Rangasamy College of Technology, Tiruchengode-637215, Tamil Nadu, India. E-mail: veerajendran@gmail.com; Fax: +91-4288-274880; Tel: +91-4288-274741-4

${ }^{b}$ Department of Chemistry, National University of Singapore, Singapore 117543, Singapore

† Electronic supplementary information (ESI) available. See DOI: 10.1039/c7ra05697d
Acalypha indica (A. indica) is a common weed that belongs to the Euphorbiaceae family. It grows in common farmlands, gardens and uncultivated lands. All parts of A. indica (leaves, root, stalk and flower), which include constituents like acalyphine, triacetoneamine, cyanogenic glucosides, and alkaloids, are highly valuable for medicinal applications, due to their antiinflammatory and antimicrobial properties. ${ }^{\mathbf{1 0}}$ The earlier studies on the extracts of Acalypha indica confirm its antimicrobial properties against pathogenic bacteria such as Escherichia coli (E. coli), Salmonella typhi (S. typhi), Pseudomonas aeruginosa (P. aeruginosa), Staphylococcus aureus (S. aureus) and Bacillus subtilis (B. subtilis). ${ }^{\mathbf{1 1} \mathbf{1 2}}$

As reported by Suresh et al., ${ }^{13}$ a GCMS study revealed that the A. indica leaf consists of 2,5-pyrrolidinedione, 1-methyl-3-omethyl-D-glucose, tetradecanoic acid, 3,7,11,15-tetramethyl-2hexadecen-1-ol, $n$-hexadecanoic acid, phytol, 9,12,15-octadecatrienoic acid, $(Z, Z, Z)$, oleic acid, 1,2-benzenedicarboxylic acid diisooctyl ester, and squalene. Along with the aforementioned compounds, Chandra Mohan et al. ${ }^{\mathbf{1 4}}$ reported that $1 \mathrm{H}$-pyrrole2,5-dione,1-ethenyl-, 3,8-nonadien-2-one, (E)-, 3,4-didehydroproline, 4-amino-3-methoxypyrazolo[3,4-d]pyrimidine, propanenitrile, and 3-(5-diethylamino-1-methoxy-3-pentynyloxy)compounds are also present in A. indica leaves.

Mosquito vectors are mainly responsible for endemic and pandemic diseases. The Ae. aegypti vector causes dengue fever in tropical and sub-tropical regions. ${ }^{1}$ An. strephensi is the 
primary vector that causes malarial infection. ${ }^{\mathbf{1 5}}$ Filariasis diseases are caused by the $C x$. quinquefasciatus vector in tropical and sub-tropical areas. ${ }^{16}$ Attempts are being made to eradicate mosquito vector borne diseases. ${ }^{17,18}$ Bio prospecting of the larvicidal properties is one of the approaches to solving the above issues. Even though different nanocomposites and chemicals provide excellent medicinal values, the properties such as non-biodegradability, bioaccumulation, bio control action, and toxicity keep them from being used for medical applications. $^{2,19}$ Among the different herbal plants, A. indica shows good antibacterial, antimalarial, parasiticide, protisticide, plasmodicide, pesticide, antimutagenic, cancer preventive properties, and hence finds wide applications as a diuretic, antifungal, purgative, antihelmintic, anti-inflammatory and in the treatment of insect bites. ${ }^{20-22}$

The medicinal applications of herbal nanoparticles with exotic textural characteristics are validated by coating these nanoparticles onto cotton fabrics. The different antimicrobial, hydrophobic and wound healing properties are explored in nanoparticles coated fabrics. ${ }^{\mathbf{2 3 , 2 4}}$ The recent studies on the selfcleaning, water repellent, high durability, antibacterial and UV protection properties, etc., of the herbal nanoparticles coated textiles strongly suggest their application in medicine. ${ }^{25,26}$

In the present study, we focus on the development of bio medically important herbal nanoparticles using the green synthesis method. The herbal nanoparticles are prepared from A. indica leaves using a ball mill without the addition of chemicals for aggregation and template shaping. In addition to the toxicity, antimicrobial and hydrophobic properties, the larvicidal properties of herbal nanoparticles are explored against three mosquito vectors, Aedes aegypti, Anopheles stephensi and Culex quinquefasciatus.

\section{Materials and methods}

\section{Collection of samples and preparation of herbal nanoparticles}

The middle leaves of $A$. indica were collected and thoroughly washed several times using deionized (D.D.) water to remove dust on the leaf surface. The leaves were then shade dried for 2 weeks. The dried leaves were ground using ball milling for $15 \mathrm{~h}$ at $300 \mathrm{rpm}$. Zirconium balls of $10 \mathrm{~mm}$ diameter were used for milling. The milling with a ball ratio of $20: 1$ was carried out for $10 \mathrm{~g}$ of leaves in a grinding jar with a zirconium protective jacket. After milling, the nanoparticles were taken for biological studies, followed by different characterization studies as reported in our previous investigations. ${ }^{27-29}$ The protocol for the preparation of herbal nanoparticles from $A$. indica leaves is shown in Fig. 1.

The X-ray diffraction patterns of the prepared $A$. indica nanoparticles (AINPs) were obtained using a powder X-ray diffractometer (XRD; X'Pert PRO, PANalytical, Almelo, the Netherlands) operated with long fine focus of the $\mathrm{Cu}$ anode at $40 \mathrm{kV}$ and $30 \mathrm{~mA}$ in Bragg-Brentano geometry. The XRD pattern was obtained in the $2 \theta$ range from $10^{\circ}$ to $80^{\circ}$ in a step-scan mode with a step size of $0.02^{\circ}$. Fourier transform infrared (FTIR) spectra of the nanoparticles were recorded using an FTIR spectrophotometer (Spectrum 100; PerkinElmer, USA) in the range of $400-4000 \mathrm{~cm}^{-1}$ using $\mathrm{KBr}$ (90 wt\% IR Grade $\mathrm{KBr}$ )

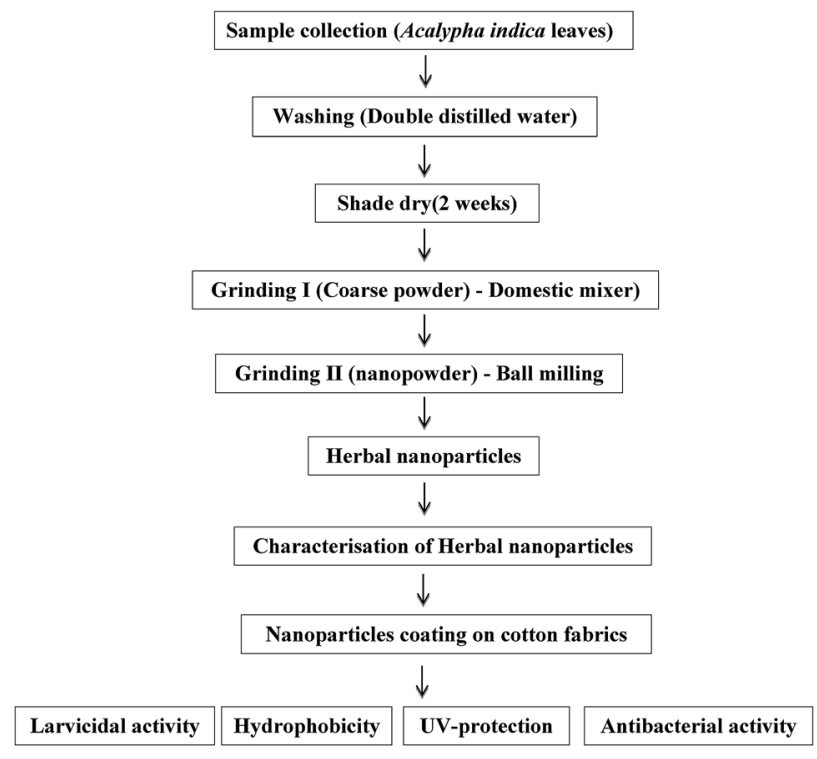

Fig. 1 Synthesis protocol for herbal nanoparticles.

matrix for making transparent disks. The green synthesized leaf nanoparticles were monitored periodically using a UV-visible (UV-vis) spectrophotometer (Agilent Cary 8454, Singapore) operated in a wide range from 180-800 nm using a step size of $5 \AA$ at different time intervals. A particle size analyzer (Nanophox, Sympatec, Germany) was used to determine the particle size distribution based on the dynamic light scattering (DLS) technique with a sub micrometer at a scattering angle of $90^{\circ}$. SEM, coupled with energy-dispersive X-ray (SEM-EDX, JSM 6360 JEOL, Japan) analysis, was used to identify the morphology, microstructure, and elemental composition of the prepared nanoparticles, fabrics coated with herbal nanoparticles and un-coated fabrics. Grain size and surface morphology of AINPs were examined through transmission electron microscopy (TEM, CM200; Philips, Eindhoven, The Netherlands) operated at a potential of $120 \mathrm{kV}$.

\section{Larvicidal activity/repellent property}

Larvae of mosquito vectors, namely, Ae. Aegypti, An. stephensi, and $C x$. quinquefasciatus, were collected from Namakkal, Tamil Nadu, India. Mosquitoes were cultured in the laboratory and were maintained continuously in laboratory conditions as reported by WHO. ${ }^{30}$ The second and fourth instar larvae were exposed to the treatment of herbal nanoparticles with different concentrations such as 100, 200, 300, 400 and $500 \mathrm{mg} \mathrm{L}^{-1}$. Each treatment was carried out in triplicate, each comprising of 25 larvae. The hatching of mosquitoes under different doses of herbal nanoparticle treatments was closely monitored from 24 to $48 \mathrm{~h}$, and the $\mathrm{LC}_{50}$ value and mortality percentage were calculated using the relation, $(T-C / 100-C) \times 100$, where $T$ is the total number of treated larvae and $C$ the total hatchability of larvae. A standard larvicidal compound, prallethrin, was used to compare the larvicidal properties of the prepared herbal nanoparticles. 


\section{In vivo toxicity}

The invertebrate animal model, with zebrafish (Danio rerio) embryos, was used to assess the acute toxicity of the herbal nanoparticles of biomedical importance. Experiments were carried out with 15 zebrafish for each dose $(12.5,25,50,75$ and $100 \mathrm{mg} \mathrm{L}^{-1}$ ) along with a control as per OECD-203 guidelines. ${ }^{31}$ All the animal model experiments were approved by the procedure of the Committee for the Purpose of Control and Supervision of Experiments on Animals (CPCSEA), as instructed by Institutional Animal Ethics Committee (IAEC) guidelines, ${ }^{32}$ K. S. Rangasamy College of Technology (Reg. no. 1826/PO/ EReBi/S/15/CPCSEA), Tiruchengode, Tamil Nadu, India. The hatching rate, mortality and developmental defects were determined for each tested dose in fish embryos, as reported in our earlier studies. ${ }^{33,34}$

\section{Coating of herbal nanoparticles}

Bleached cotton fabric $\left(100 \%\right.$, mass $138.84 \mathrm{~g} \mathrm{~m}^{-2}, 116$ ends per inch, 84 picks per inch) was used as a substrate for coating the herbal nanoparticles through a padding mangle at a rate of $35 \mathrm{rpm}$ for $5 \mathrm{~min}$ to collect the uniformly coated fabrics ${ }^{35,36}$ in order to explore the hydrophobicity and UV-protection properties. Then, the coated fabrics were studied for their enhanced physico-mechanical and antimicrobial properties such as coating thickness, tensile and tear strength, crease recovery angle, air permeability and bacterial growth reduction; these data are given in detail in the ESI file (S1). $\dagger$

\section{Hydrophobicity and UV-protection properties}

Hydrophobicity and UV-protection properties of herbal nanoparticles were examined for self-cleaning and UV radiation protection applications, respectively. The hydrophobic nature of the herbal nanoparticle treated and untreated fabrics was ascertained based on the water contact angle (VCA Optima, ACT Product Inc., Japan). Digital photographic analysis of water droplets placed on the fabric surface was conducted before washing and after the $10^{\text {th }}$ wash. Similarly, UV resistant properties of herbal nanoparticles were also studied on the nanoparticle coated fabrics using UV transmission spectra (Lambda 35; PerkinElmer, USA), in the wavelength range of $280-400 \mathrm{~nm}$ as per the ASTM D6603 standard.

\section{Antimicrobial studies}

Two bacterial cultures, namely, Gram-positive $S$. aureus (ATCC 6538P) and Gram-negative E. coli (ATCC 9677), were obtained from the National Collection of Industrial Microorganisms (NCIM) (National Chemical Laboratory, Pune, India). The obtained bacterial cultures were further sub-cultured several times at $37^{\circ} \mathrm{C}$ for $24 \mathrm{~h}$. Inoculation of a loopful of test organisms into nutrient broth was carried out to prepare fresh bacterial inoculums, and then incubated at $37^{\circ} \mathrm{C}$ for $5-8 \mathrm{~h}$ till a fair turbidity was obtained. Fresh cultures were swabbed on a nutrient agar plate and then herbal particles of different concentrations (25, 50 and $\left.100 \mathrm{mg} \mathrm{mL}^{-1}\right)$ were loaded onto the well punctured in the nutrient agar plate. After $24 \mathrm{~h}$ of incubation, zones of inhibition around the herbal nanoparticles loaded onto the agar well were measured using a millimeter ruler.

\section{Results and discussion}

\section{Characterization}

Herbal nanoparticles of AINPs were synthesized and comprehensively characterized for evaluating the influence of nanoscale particles on the medicinal, antimicrobial and functional properties. The XRD pattern of AINPs is shown in Fig. 2, which confirms the absence of diffraction peaks i.e., amorphous nature, except for the observed broad band at $2 \theta$ values $\left(20-30^{\circ}\right)$. Generally, the amorphous nature of the particles is non-toxic to living organisms and hence, amorphous herbal nanoparticles enhance the biocompatibility for clinical applications.

The FTIR spectrum obtained from AINPs is shown in Fig. 3. The peaks observed between $3200 \mathrm{~cm}^{-1}$ and $3500 \mathrm{~cm}^{-1}$ are assigned to the presence of superficially absorbed water and the stretching mode of the $\mathrm{OH} / \mathrm{NH}$ group, respectively. The bands observed at $2920 \mathrm{~cm}^{-1}$ and $2858 \mathrm{~cm}^{-1}$ correspond to the stretching vibrations of aliphatic and aromatic $\mathrm{C}-\mathrm{H}$ bonds in the nanoparticles. ${ }^{37}$ The peak at $1626 \mathrm{~cm}^{-1}$ is identified as the

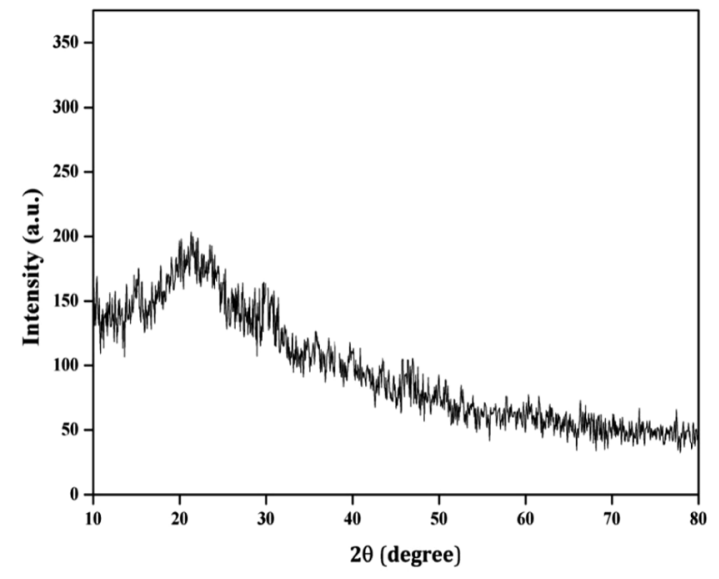

Fig. 2 XRD spectra of AINPs.

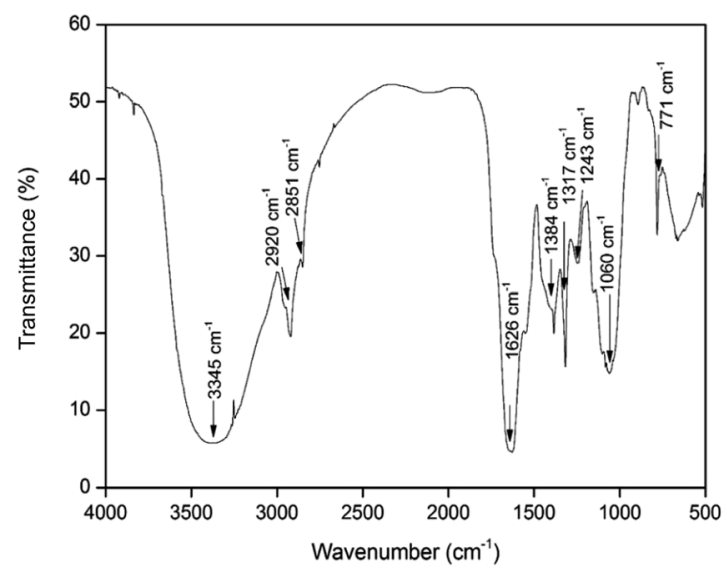

Fig. 3 FTIR spectrum of $A$. indica leaf nanoparticles. 
carbonyl $(\mathrm{C}=\mathrm{O})$ stretching vibration in the amide linkage of the plant protein. ${ }^{38}$ The band at $1384 \mathrm{~cm}^{-1}$ is assigned to the primary amine $(\mathrm{N}-\mathrm{H})$ bending mode, which is the appropriate characteristic peak of flavanones and terpenoids present in $A$. indica plant leaves. ${ }^{39}$ The peaks at $1317 \mathrm{~cm}^{-1}$ and $1243 \mathrm{~cm}^{-1}$ signify the carboxylic acid (C-O) group ${ }^{40}$ and the obtained peak at $1060 \mathrm{~cm}^{-1}$ discloses the $\mathrm{C}-\mathrm{N}$ stretching vibrations of aliphatic amines. The band observed at $660 \mathrm{~cm}^{-1}$ is due to the

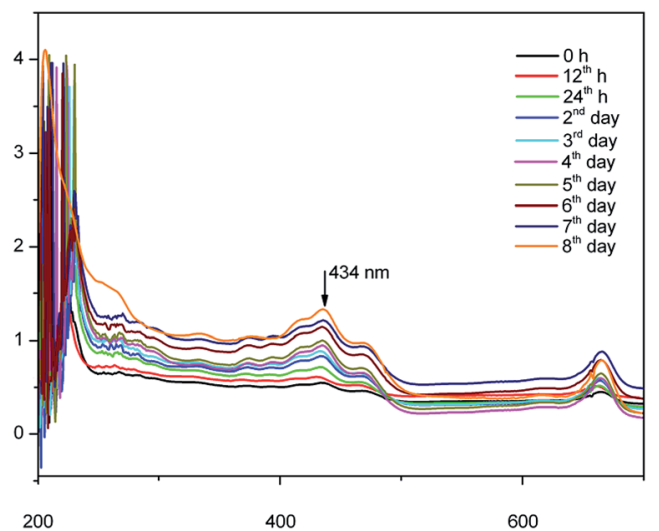

Fig. 4 UV-visible spectrum of $A$. indica leaf nanoparticles in water.

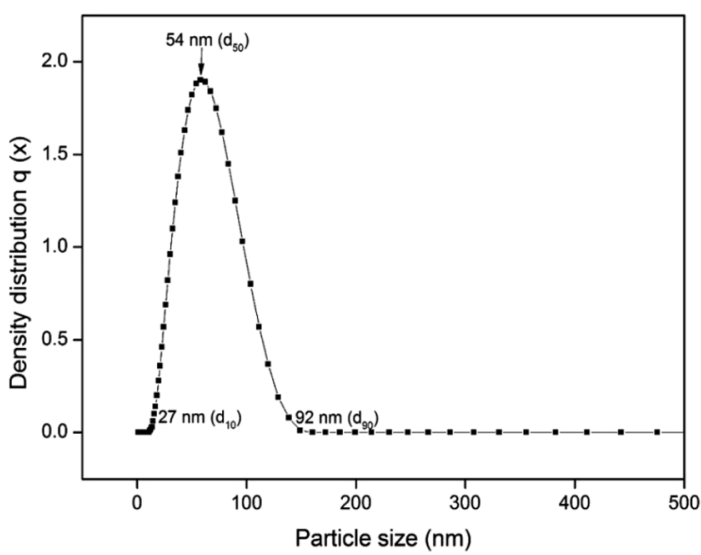

Fig. 5 Particle size distribution of $A$. indica leaf nanoparticles. deformation of $\alpha$-glucopyranose rings of carbohydrates. ${ }^{40,41}$ The FTIR spectrum confirms the presence of major plant compounds that are responsible for the antimicrobial, larvicidal and UV-protection properties.

Fig. 4 shows the UV-vis spectrum of the synthesized herbal AINPs dispersed in water. In the present study, $A$. indica has an absorbance in the UV region at around $434 \mathrm{~nm}$, which favours the anti-reflective and UV-adsorption properties for textile applications. Similar to silver nanoparticles, the plant based nanomaterials also show antimicrobial activity, but with lower toxicity. To determine the stability of the nanoparticles, we obtained the UV-vis spectra of the leaf nanoparticles at different time intervals for 8 days. It is interesting to see from Fig. 4 that there is no difference in absorption spectra, which confirms the higher stability of $A$. indica nanoparticles. The antireflective properties of the nanoparticles are favorable for developing UV-resistant biomaterials in an eco-friendly way. Fig. 5 shows the particle size distribution curve of the prepared AINPs. The average particle size of the prepared nanoparticles is around $54 \pm 3 \mathrm{~nm}$.

SEM and TEM images of the AINPs are shown respectively in Fig. 6 and 7. The topographical characterization of the nanoparticles observed in the SEM image shows that the herbal AINPs are uniform in structure. The SEM image shows the discrete distribution of herbal nanoparticles at higher magnification. The elemental composition of AINPs analyzed using EDX shows $\mathrm{C}$ and $\mathrm{O}$ peaks corresponding to elements such as $\mathrm{Na}, \mathrm{Mg}, \mathrm{Si}, \mathrm{Cl}$, $\mathrm{K}$, and $\mathrm{Ca}$ metal ions, which confirm the inorganic compounds. A similar observation was reported in our previous study on obtaining the herbal nanoparticles from Tridax procumbens under different milling periods. ${ }^{26}$ The TEM (Fig. 7) image of the prepared AINPs confirms the spherical nature with high dispersion. The average particle size of the herbal AINPs obtained from the TEM image is about $50 \mathrm{~nm}$. This is in close agreement with the measured particle size $(54 \mathrm{~nm})$ of the AINPs from the particle size distribution measurements.

\section{Larvicidal activity}

The larvicidal activities of the five different concentrations of AINPs to Aedes aegypti, Anopheles stephensi and Cx. quinquefasciatus are shown in Table 1. After $48 \mathrm{~h}$ of exposure, the AINPs were tested for their larvicidal activities according to the
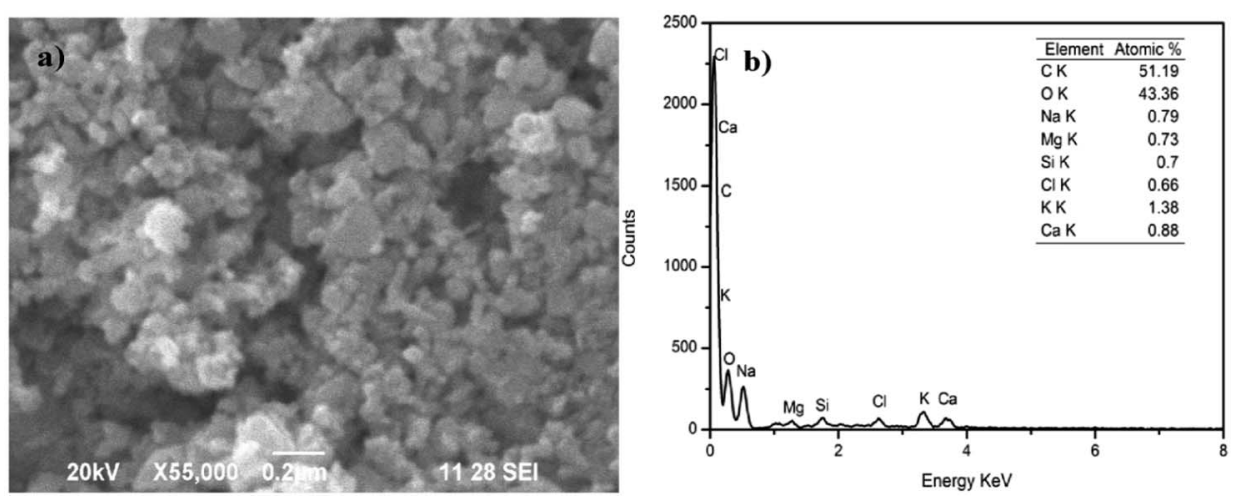

Fig. 6 Particle size distribution of $A$. indica leaf nanoparticles (a) SEM image and (b) EDX analysis. 


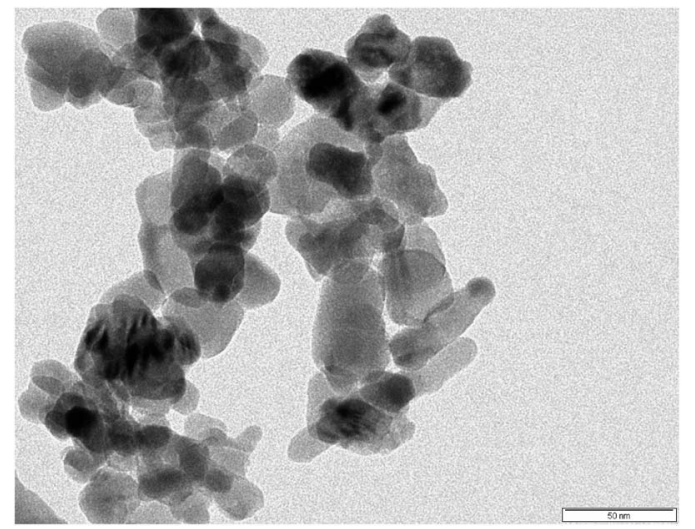

Fig. 7 TEM image of $A$. indica nanopowders.

treatment concentrations. The larvicidal activities of the herbal nanoparticles were found to be high in terms of mortality rate at a higher concentration of AINPs against Aedes aegypti, Anopheles stephensi and Cx. Quinquefasciatus $\left(\mathrm{LC}_{50}=500 \mathrm{mg} \mathrm{L}^{-1}\right)$, as shown in Table 1. However, lower mortality was observed while exploiting both the $\mathrm{I}^{\text {st }}$ and $\mathrm{IV}^{\text {th }}$ instars, due to the low amount of AINPs against these three mosquito vectors.

Several Euphorbiaceae plant extracts are known to exhibit larvicidal activity against these three mosquito vectors. ${ }^{42}$ Previously, extracts of A. indica using methanol, ethyl acetate, benzene and chloroform were studied for their larvicidal and ovicidal activities against Anopheles stephensi. In fact, crude nanoparticles of $A$. indica revealed comparatively higher larvicidal action than the extracted compounds of $A$. indica (by 15 percent), against the three mosquito vectors. ${ }^{43}$ The observed enhanced larvicidal activities are due to the exposure of the highly reactive surface area of the herbal AINPs. It is inferred from the present study that the obtained crude herbal nanoparticles with high surface areas act as an effective mosquito repellent and larvicide. The effectiveness of the prepared herbal A. indica nanoparticles was compared with a commercially used larvicide, prallethrin, with the least effective concentrations of A. indica nanopowder against mosquito larvae $\left(120 \mathrm{mg} \mathrm{L}^{-1}\right)$. Table 1 shows similar larvicidal properties for Prallethrin and prepared herbal nanoparticles. There are reports that show that Prallethrin is a poisonous material at lower concentration $\left(25 \mathrm{mg} \mathrm{mL}{ }^{-1}\right) \cdot{ }^{44}$ Our study shows that the use of herbal nanoparticles with good larvicidal action is amenable for mosquito control as an alternative larvicide to synthetic chemicals.

\section{In vivo toxicity}

The toxicity of AINPs was explored to determine the safe use for humans and other animals. Most herbs are non-toxic and good for health, due to the presence of numerous organic compounds. In order to explore the toxicity level of the nanoparticles, zebrafish embryos were treated with the AINPs and their developmental stages were monitored for $72 \mathrm{~h}$ as shown in Table 2. Among the five different concentrations of nanoparticles, particles of $200 \mathrm{mg} \mathrm{L}{ }^{-1}$ concentration were highly effective with no mortality of the zebrafish embryos. In addition, a high concentration of nanoparticles does not affect the embryogenesis or hatching rate. Interaction of herbal particles associated with the biocompatibility of the plant compounds does not affect the fish developmental stages.

A study investigating several metal oxide nanoparticles for their acute toxicity studies revealed significant toxicity at higher concentration when the embryos were tested for $72 \mathrm{~h} .{ }^{45}$ Our observation reveals the non-toxic nature of the prepared AINPs against embryos, due to the samples being devoid of any processing chemicals like polar and non-polar solvents. On comparison of AINPs with other nano metal oxides, the herbal nanoparticles were found to be highly biocompatible with invertebrate animal model zebrafish by means a manifold reduction in mortality. Unfortunately, exposure concentrations of herbal nanoparticles are different from other metal oxide nanoparticles, ${ }^{41}$ since herbal nanoparticles are of biological

Table 2 In vivo toxicity of herbal nanoparticles used to treat zebrafish

\begin{tabular}{lllll}
\hline & & \multicolumn{3}{l}{ Mortality $(\%)$} \\
\cline { 4 - 5 } Nanoparticles & $\begin{array}{l}\text { Concentrations } \\
\left(\mu \mathrm{g} \mathrm{mL}^{-1}\right)\end{array}$ & $24 \mathrm{~h}$ & $48 \mathrm{~h}$ & $72 \mathrm{~h}$ \\
\hline A. indica & Control & 0 & 0 & 0 \\
& 25 & 0 & 0 & 3.3 \\
& 50 & 0 & 3.3 & 6.6 \\
& 75 & 0 & 6.6 & 13.3 \\
& 100 & 0 & 6.6 & 13.3 \\
Prallethrin & 200 & 0 & 6.6 & 6.6 \\
& 25 & 100 & 0 & 0
\end{tabular}

Table 1 Larvicidal activity of herbal nanoparticles against Ae. Aegypti, An. Stephensi and Cx. Quinquefasciatus

A. indica nanoparticles at different concentrations (ppm)

Prallithrin

\begin{tabular}{|c|c|c|c|c|c|c|c|}
\hline Mosquito species & Instar & $\left(120 \mathrm{mg} \mathrm{L}^{-1}\right)$ & $100 \mathrm{mg} \mathrm{L}^{-1}$ & $200 \mathrm{mg} \mathrm{L}^{-1}$ & $300 \mathrm{mg} \mathrm{L}^{-1}$ & $400 \mathrm{mg} \mathrm{L}^{-1}$ & $500 \mathrm{mg} \mathrm{L}^{-1}$ \\
\hline \multirow[t]{2}{*}{ Ae. aegypti } & I & $100.0 \pm 0.00$ & $16.07 \pm 2.71$ & $33.12 \pm 1.41$ & $64.43 \pm 3.17$ & $97.15 \pm 2.32$ & $100.0 \pm 0.00$ \\
\hline & IV & $100.0 \pm 0.00$ & $8.11 \pm 3.11$ & $25.14 \pm 2.04$ & $47.03 \pm 2.10$ & $93.03 \pm 1.15$ & $100.0 \pm 0.00$ \\
\hline \multirow{3}{*}{ Cx. Quinquefasciatus } & IV & $100.0 \pm 0.00$ & $9.32 \pm 2.05$ & $27.16 \pm 1.74$ & $52.11 \pm 1.76$ & $94.01 \pm 3.02$ & $100.0 \pm 0.00$ \\
\hline & I & $100.0 \pm 0.00$ & $19.07 \pm 3.12$ & $38.05 \pm 2.72$ & $69.32 \pm 2.43$ & $100.0 \pm 0.11$ & $100.0 \pm 0.00$ \\
\hline & IV & $100.0 \pm 0.00$ & $9.32 \pm 2.05$ & $26.42 \pm 1.65$ & $54.27 \pm 1.54$ & $96.01 \pm 0.31$ & $100.0 \pm 0.00$ \\
\hline
\end{tabular}


origin and need to be tested at higher concentrations. Even at higher concentrations, the particles do not cause significant toxicity, which deserves wider use of the AINPs in the biomedical field for multidisciplinary applications as antimicrobial, mosquito repellent and biocompatible materials. On comparing the larvicidal activity of $A$. indica nanoparticles with the commercially used larvicide, prallethrin, it was observed that the synthetic oil prallethrin was highly toxic, since it showed $100 \%$ mortality at $24 \mathrm{~h}$. From the aforementioned study, it can be said that $A$. indica nanoparticles can be used as a natural larvicide in place of prallethrin, due to its lower toxicity and better larvicidal properties.

The surface characteristics of the AINPs like hydrophobicity and adherence properties were explored for biomedical applications, using cotton fabric. The ultraviolet protection factor (UPF) in the wavelength ranges from 280 to $400 \mathrm{~nm}$ was studied after coating the herbal particles onto the fabric, and the results are shown in Table 3. The percentage of transmittance for UV-A and UV-B is almost similar to that of the untreated fabrics. ${ }^{46} \mathrm{~A}$ significant reduction in transmittance was observed for herbal nanoparticle coated fabrics, which is ascribed to the blocking of UV-B and UV-A radiation due to the coating. In addition, the percentage blocking of UV-B radiation by the herbal nanocomposite is high (57\%) as compared to that of chitosancoating. ${ }^{47}$ Furthermore, the resistance rate of UV radiation for the herbal nanocomposite after the $5^{\text {th }}$ and $10^{\text {th }}$ washes is moderately reduced (4\%) in herbal AINPs treated fabrics compared to that of the untreated cotton fabrics. On the basis of the ASTM D6603 standard data, the UPF value for the fabrics is more than $50 \%$, demonstrating better protection of fabric from UV rays. The calculated UPF value for the AINPs coated fabrics was observed to be $57.7 \pm 0.06$, i.e., higher than the limitation (i.e., 50) ascribed to the higher resistance to UV irradiation. However, the values for un-coated and chitosan-coated fabrics exhibit lesser UV protection $(<50)$ compared to those of the herbal nanocomposite coated fabrics. Thus, the herbal nanoparticles have an increment of nearly $50 \%$ in UV protection, compared to the un-coated fabric. This is attributed to the

Table 3 UV protection and water repellent properties of un-coated, chitosan and herbal nanocomposite coated fabric samples

Sample names

UPF value

Contact angle

Before wash

Un-coated fabrics

Chitosan

$13.9 \pm 0.63$

$42.8 \pm 0.46$

Nanocomposite

$57.7 \pm 0.06$

0

$119 \pm 1$

$151 \pm 3$

After $5^{\text {th }}$ wash

Un-coated fabrics

$11.2 \pm 0.93$

Chitosan

Nanocomposite

$40.1 \pm 0.77$

$55.6 \pm 0.70$

0

$101 \pm 3$

$135 \pm 3$

After $10^{\text {th }}$ wash

Un-coated fabrics

$10.8 \pm 0.61$

Chitosan

Nanocomposite
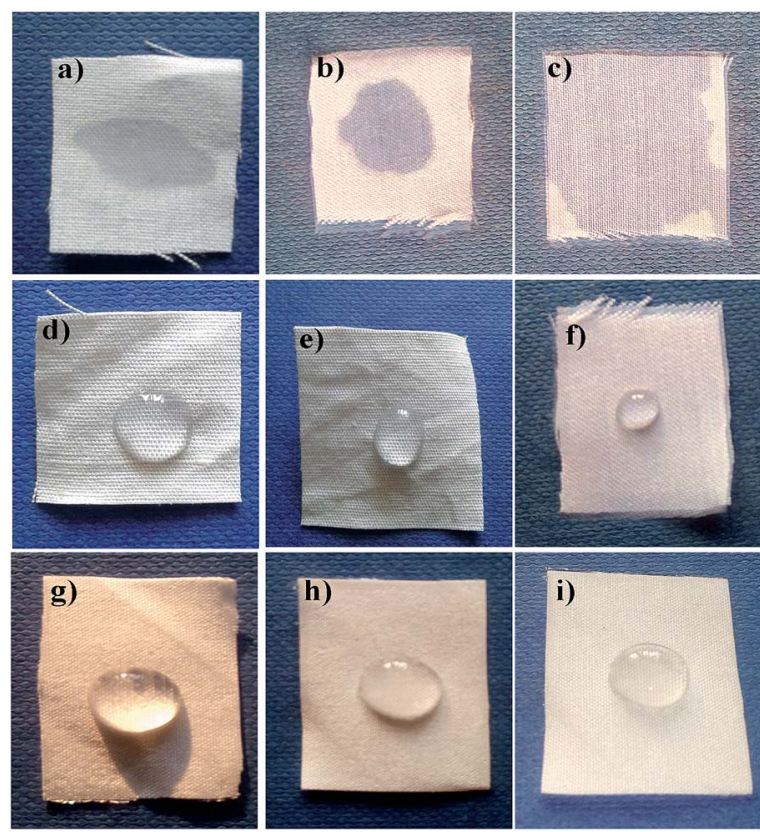

i)

Fig. 8 Photographic images of water droplets placed on un-coated and coated fabrics before and after washing. (a) Un-coated, (b and c) un-coated $5^{\text {th }} \& 10^{\text {th }}$ wash, (d) C-CF, (e and f) C-CF $5^{\text {th }} \& 10^{\text {th }}$ wash, (g) $\mathrm{Al}-\mathrm{C}-\mathrm{CF}$, (h and i) Al-C-CF $5^{\text {th }} \& 10^{\text {th }}$ wash

ability of herbal nanoparticles to provide protection from UVrays, which would be an additional advantage for developing radiation protective clothing. ${ }^{48}$

\section{Hydrophobic activity}

The water repellent property of the particles is one of the most interesting and desired properties of medical textiles, especially in pharmaceuticals and biomedicine. ${ }^{4748}$ Researchers have reported that the hydrophobic or water repellent property is a function of the textile surface morphology and reduced surface energy. ${ }^{\mathbf{4} 49-51}$ In our studies, we have investigated the water repellent efficiency of herbal nanoparticles, in terms of contact angle, by coating the herbal AINPs onto cotton fabrics. Generally, the fabrics with angle greater than $90^{\circ}$ are considered to be hydrophobic, while those with angle greater than $150^{\circ}$ are
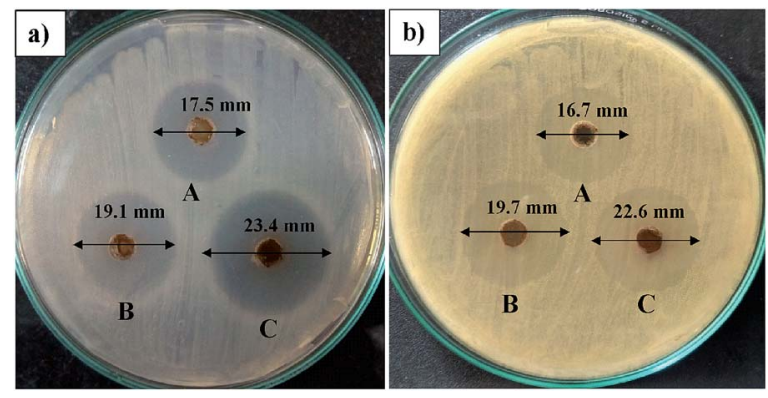

Fig. 9 Antimicrobial activity of $A$. indica nanoparticles for different concentrations: (A) $25 \mathrm{mg} \mathrm{mL}^{-1}$, (B) $50 \mathrm{mg} \mathrm{mL}^{-1}$ and (C) $100 \mathrm{mg} \mathrm{mL}^{-1}$ against $E$. coli and $S$. aureus. 
Table 4 Antimicrobial activity of herbal nanoparticles

\begin{tabular}{llll}
\hline & & \multicolumn{2}{c}{ Concentrations of AINPs, (zone of inhibition (mm)) } \\
\cline { 3 - 4 } Herbal nanoparticles & Test organisms & $25 \mathrm{mg} \mathrm{mL}$ & $50 \mathrm{mg} \mathrm{mL}^{-1}$ \\
\hline A. indica & E. coli & $17.51 \pm 0.23$ & $19.14 \pm 0.12$ \\
& S. aureus & $16.72 \pm 0.21$ & $19.71 \pm 0.12$
\end{tabular}

superhydrophobic. From Table 3, it is seen that herbal AINPs coated cotton fabrics show higher contact angle $\left(151^{\circ}\right)$ than the standard value of superhydrophobic materials. This in turn confirms the superhydrophobic behaviour of $A$. indica coated on the fabrics. The contact angle of un-coated, chitosan and nanocomposite coated cotton fabrics remains the same even after the $5^{\text {th }}$ and $10^{\text {th }}$ washes. This is due to the strong adherence of the particles and thereby the retention of the water repellent property even after 10 washes. It is interesting to note that the coating of herbal nanoparticles renders a superhydrophobic nature $\left(151^{\circ}\right)$ to the fabrics, thereby favoring selfcleaning applications.

From the micrograph (Fig. 8), it is further evident that the chitosan and nanoparticle coated cotton fabrics have higher contact angles than the un-coated and the chitosan coated fabrics, owing to higher water repellent properties of the chitosan nanocomposite coated fabrics. In contrast, the liquid droplet on the un-coated fabric immediately seeps into the fabric, due to the very low contact angle and microspores in the materials, which make the materials more hydrophilic. ${ }^{47,49}$ This might also be attributed to the fact that the improved hydrophobicity of the fabrics is the result of the change in the surface morphology and surface energy, due the coating of nanomaterials, ${ }^{52}$ which is correlated with our observation for the superhydrophobic nature of the herbal nanoparticles.

\section{Antimicrobial activity}

The screening of the antibacterial activities of the prepared AINPs was carried out based on the zone of inhibition observed at different concentrations, namely 25,50 and $100 \mathrm{mg} \mathrm{mL}^{-1}$ of AINPs (Fig. 9) and is summarized in Table 4. The agar well loaded with AINPs shows the maximum zone of inhibition against $E$. coli $(23.5 \mathrm{~mm})$ and $S$. aureus $(22.7 \mathrm{~mm})$ at a concentration of $100 \mathrm{mg} \mathrm{mL}^{-1}$. The magnitude of the inhibition zone is slightly higher in E. coli than $S$. aureus. In the case of treatment with the low concentration ( $25 \mathrm{mg} \mathrm{mL}^{-1}$ ) of AINPs, the inhibition zone was found to be $17.51 \pm 0.23$ and $16.72 \pm 0.21 \mathrm{~mm}$, respectively, against $E$. coli and $S$. aureus.

A previous report on the aqueous extract of $A$. indica demonstrates the inhibition zone of $9 \mathrm{~mm}$ against $E$. coli and no inhibition zone against $S$. aureus. ${ }^{53}$ Similarly, acetone aqueous extract from AINPs shows the minimum antimicrobial properties against $S$. aureus $(22 \mathrm{~mm})$ and E. coli $(15 \mathrm{~mm}) .{ }^{54}$ Quantitative evaluation of the antimicrobial activity of herbal nanoparticle coated fabrics for medical textile applications was also conducted, which is included with these results as a ESI file (S1). $\uparrow$ The observed antimicrobial results of the tested herbal nanoparticles are comparatively higher than the organic extracts of the particles. This is due to the existence of intact reactive organic compounds such as acalyphine, triacetoneamine, cyanogenic glucosides, and alkaloids ${ }^{\mathbf{1 0}}$ that are responsible for biochemical and cell wall reactions to inhibit bacterial growth. This is one of the superior properties of dry herbal nanoparticles possessing multifunctional characteristics like mosquito repellency, antimicrobial properties, and superhydrophobicity.

\section{Conclusion}

Herbal nanoparticles synthesized from A. indica leaves were screened and evaluated for their improved physico-mechanical and biological properties to explore their possible applications in biomedicine. The superhydrophobic nature and excellent UVprotection properties of the herbal particles are additionally proven to be favourable at higher concentrations (up to $200 \mu \mathrm{g} \mathrm{mL}^{-1}$ ) for medical textiles and self-cleaning applications. Larvicidal activity against major disease mosquito vectors is well demonstrated in the present investigation, which aids in the development of eco-friendly nanoparticles of biomedical importance. The prepared herbal nanoparticles exhibit exotic in vitro and in vivo biocompatibility, and antimicrobial activity that enhance the application of cost effective herbal nanoparticles as potent biomaterials to remediate life threatening problems in healthcare.

\section{Conflicts of interest}

Authors have no conflicts to declare.

\section{Acknowledgements}

The authors acknowledge the financial support provided by Board of Research and Nuclear Science (BRNS), Mumbai (Sanction no: 2013/34/30/BRNS/1127 dt.19.9.2013). One of the authors (Dr R. S.) is thankful to the University Grants Commission (UGC), New Delhi for the award of Post-Doctoral Fellowship for Women (F.15-1/2015-17/PDFWM-2015-17-TAM36274 dt.12/10/2015).

\section{References}

1 S. Vijaya kumar, P. Mani, T. M. M. John Bastin and G. Ravikumar, Int. J. Med. Biosci., 2012, 1(3), 33-41.

2 S. Pawel, M. Magdalena and M. Elzbieta, Nano, 2011, 6, 509539. 
3 A. Nasrollahi, K. Pourshamsian and P. Mansourkiaee, Int. J. Nano Dimens., 2011, 1, 233-239.

4 N. R. Dhineshbabu, P. Manivasakan, A. Karthik and V. Rajendran, RSC Adv., 2014, 4, 32161-32173.

5 S. C. Mccombie, Soc. Sci. Med., 1996, 43, 933-945.

6 Q. Daoming and N. K. Peter, Drugs R\&D, 2003, 4, 1-18.

7 N. G. Das, I. Baruah, P. K. Talukdar and S. C. Das, J. Vector Borne Dis., 2003, 40, 49-53.

8 K. Murugan, P. Murugan and A. Noortheen, Bioresour. Technol., 2007, 98, 198-201.

9 B. Gabriela, L. A. Ioana, B. Nicoleta, O. Cristina and M. Aurelia, Ind. Crops Prod., 2015, 67, 18-24.

10 M. A. Rahman, C. B. Sitesh and R. Mohammed, Pak. J. Pharm. Sci., 2010, 23, 256-258.

$11 \mathrm{H}$. Pao-Chuan, M. Jeng-Leun and H. Shu-Hui, Food Microbiol., 2001, 18, 35-43.

12 A. Zahir Hussain and S. Kumaresan, Asian J. Plant Sci. Res., 2013, 3, 46-49.

13 M. Suresh, S. A. A. Mohammad, K. R. Pradipta, A. Panneerselvam and N. Thajuddin, Asian Pac. J. Trop. Biomed., 2016, 6(3), 185-191.

14 S. Chandra Mohan, S. Dinakar, T. Anand, R. Elayaraja and B. S. Priya, Int. J. PharmTech Res., 2012, 4(3), 1050-1054.

15 R. Borah, M. C. Kalita, A. Kar and A. K. Talukdar, Afr. J. Biotechnol., 2010, 9, 2527-2530.

16 E. J. Muturi, P. Burgress and R. J. Novak, Am. J. Trop. Med. Hyg., 2008, 78, 536-537.

17 R. Maheswaran, S. Sathish and S. Ignacimuthu, Int. J. Integr. Biol., 2008, 2, 214-217.

18 M. Pavunjarj, K. Baskar, V. Duraipandiyan, N. Abdullah AlDhabi, V. Rajendran and G. Benelli, J. Cluster Sci., 2017, 1-16.

19 R. Suriyaprabha, K. Gopalu, P. Muthusamy, Y. Rathinam, V. Rajendran and N. Kannan, RSC Adv., 2014, 4, 8461-8465.

20 Y. M. Shivakar and V. L. Kumar, Pharm. Biol., 2003, 41, 263265.

21 M. Govindarajan, A. Jebanesan, D. Reetha, R. Anisath, T. Pushpanathan and K. Samidurai, Eur. Rev. Med. Pharmacol. Sci., 2008, 12, 299-302.

22 P. Prasad and M. Estari, International Interdisciplinary Research Journal, 2014, 4, 175-182.

23 S. Sheila and W. Jakub, Antimicrob. Agents, 2012, 19, 387-406.

24 B. Venkatrajah, V. Vanitha Malathy, B. Elayarajah, S. Mohan, R. Rajendren and R. Rammohan, J. Med. Sci., 2012, 12, 148160.

25 R. Rajendran, R. Radhai, T. M. Kotresh and C. Emilia, Carbohydr. Polym., 2013, 91, 613-617.

26 N. R. Dhineshbabu, P. Manivasakan, R. Yuvakkumar, P. Prabu and V. Rajendran, J. Nanosci. Nanotechnol., 2013, 13, 4017-4024.

27 M. Vinoth, R. SuriyaPrabha, S. Arunmetha, A. Karthik, S. Karthik, P. Paramasivam, P. Prabu, P. Manivasakan, K. Saminathan and V. Rajendran, Synth. React. Inorg., Met.Org., Nano-Met. Chem., 2015, 46, 1445-1449.

28 S. Karthik, R. Suriyaprabha, K. S. Balu, P. Manivasakan and V. Rajendran, IET Nanobiotechnol., 2016, 11, 12-17.
29 S. Karthik, M. Vinoth, K. S. Balu, R. Suriyaprabha, P. Manivasakan, V. Rajendran and V. Suresh, J. Alloys Compd., 2017, 723, 698-707.

30 K. Baskar, V. Sudha, G. Nattudurai, S. Ignacimuthu, V. Duraipandiyan, M. Jayakumar, N. Abdullah Al-Dhabi and G. Benelli, Physiol. Mol. Plant Pathol., 2017, 30, 1-5.

31 OECD, Guidelines for the Testing of Chemicals, No. 203: Fish Acute toxicity Test, 1992, Adopted 17/07/1992.

32 K. B. Dinesh and C. Desai, Indian J. Pharmacol., 2014, 46(3), 257-265.

33 M. Prabhu, R. Suriyaprabha, V. Rajendran, P. Kulandaivelu and S. Valiyaveettil, RSC Adv., 2014, 4, 43630-43640.

34 K. Kavitha, W. Chunyan, D. Navaneethan, V. Rajendran, S. Valiyaveettil and A. Vinoth, RSC Adv., 2014, 4, 4395143961.

35 B. Gupta, S. Saxena and A. Arora, J. Fibre Text. Res., 2011, 36, 272-280.

36 S. S. Ugur, M. Sarısik and A. H. Aktas, Nanotechnology, 2010, 21, 325603-325610.

37 P. Prakash, P. Gnanaprakasam, R. Emmanuel, S. Arokiyaraj and M. Saravanan, Colloids Surf., B, 2013, 108, 255-259.

38 K. Rajathi and S. Sridhar, International Journal of Green Chemistry and Bioprocess, 2012, 2, 39-43.

39 B. Priya, S. Mantosh, M. Aniruddha and D. Papita, Bioresources and Bioprocessing, 2014, 1, 1-10.

40 P. Logeswari, S. Silambarasan and J. Abraham, Sci. Iran., 2013, 20, 1049-1054.

41 P. Saravanan, G. Chandramohan, J. Mariajancyrani and P. Shanmugasundaram, Int. J. Environ. Sci., 2013, 2, 1-5.

42 A. A. Rahuman, G. Gopalakrishnan, P. Venkatesan and K. Geetha, Parasitol. Res., 2008, 102, 867-873.

43 M. Govindarajan, A. Jebanesan, T. Pushpanathan and K. Samidurai, Parasitol. Res., 2008, 103, 691-695.

44 M. B. Emmanuel, S. Moorthy, G. Ganeshwala and G. Abraham, J. Med. Toxicol., 2010, 6, 7-30.

45 L. C. Wehmas, C. Anders, J. Chess, A. Punnose, C. B. Pereira, J. A. Greenwood and R. L. Tanguay, Toxicol. Rep., 2015, 2, 702-715.

46 S. Agnihotri, S. Mukherji and M. Suparna, $R S C A d v ., 2014,4$, 3974-3983.

47 N. F. Attia, M. Moussa, A. M. F. Sheta, R. Taha and H. Gamal, Prog. Org. Coat., 2017, 106, 41-49.

48 N. F. Attia, M. Moussa, A. M. F. Sheta, R. Taha and H. Gamal, Prog. Org. Coat., 2017, 104, 72-80.

49 K. Autumn, Y. A. Liang, S. T. Hsieh, W. Zesch, W. P. Chan, T. W. Kenny, R. Fearing and R. J. Full, Nature, 2000, 405, 681-685.

50 S. Wang, C. Liu, G. Liu, M. Zhang, J. Li and C. Wang, Appl. Surf. Sci., 2011, 258, 806-810.

51 A. Nakajima, K. Hashimoto and T. Watanabe, Monatsh. Chem., 2001, 132, 31-41.

52 B. Liu, L. Wang, Y. Gao, T. Tian, J. Min, J. Yao, Z. Xiang, C. Huang and C. Hu, Text. Res. J., 2015, 85, 795-803.

53 S. S. Ugur, M. Sarısik and A. H. Aktas, Nanotechnology, 2010, 21, 325603-325610.

54 P. Mohanpuria, K. N. Rana and S. K. Yadav, J. Nanopart. Res., 2008, 10, 507-517. 